\title{
An Intelligent Binocular Vision System with Zoom Lens for 3D Scene Reconstruction
}

\author{
Shuyang Dou ${ }^{1 *}$, Hiroshi Nagahashi ${ }^{2}$ \\ ${ }^{1}$ Tokyo Institute of Technology, Department of Information Processing, 4259 Nagatsuta-cho, Midori-ku, \\ Yokohama, Kanagawa, Japan, 226-8503. \\ 2 Tokyo Institute of Technology, Laboratory for Future Interdisciplinary Research of Science and Technology \\ (FIRST), 4259 Nagatsuta-cho, Midori-ku, Yokohama, Kanagawa, Japan, 226-8503.
}

* Corresponding author. Email: dsy.dlut@gmail.com

Manuscript submitted November 5, 2016; accepted December 23, 2016.

doi: 10.17706/ijcee.2016.8.6.304-318

\begin{abstract}
In this paper, we propose an intelligent binocular vision system for 3D scene reconstruction by using zoom lens. Unlike many existing methods which use precalibrated fixed focal length lens, our system allows users to change zoom and focus settings accordingly while taking photos. Thus, more details about some interesting parts of a scene can be captured clearly. In addition, using a zoom lens is advantageous especially when the system can only be moved within some small areas. After changing camera's zoom or focus setting, its intrinsic parameters will be changed. It is important to calibrate these parameters accurately in order to reconstruct the 3D scene model successfully. We propose an efficient camera calibration method which can dynamically compute the focal length and principle point. It is proved that this method could generate more accurate estimations than the linear interpolation method. We introduced a criterion in our system which can control the zoom lens properly. Our system also contains a strategy to detect and remove redundant photos, which can speed up the computation. Experimental results show that our proposed system could create more plausible 3D models.
\end{abstract}

Key words: Binocular vision system, camera calibration, Zoom lens, 3D scene reconstruction.

\section{Introduction}

Reconstructing the 3D model of a scene is an important problem in Computer Vision (CV) field. Though enormous efforts have been invested into this problem since the last decades, it is still considered to be one of the most challenging problems in CV community. This problem is closely related to many other research problems, like structure from motion (SfM) [1], simultaneous localization and mapping (SLAM) [2], pattern recognition, reverse engineering, augmented reality, etc. Recently, the research of autonomous car has gained a lot of focus. One of the significant subjects related to this research is the real-time reconstruction of 3D model of surrounding areas.

By far, a lot of researchers have proposed many sophisticated approaches to this problem. These approaches can be roughly divided into two groups: Firstly, the active group, which directly measure the depth information using some special sensors, like a laser scanner [3], a Time of Flight (ToF) camera [4], etc. Secondly, the passive group, which usually estimate the depth information from image correspondences, like stereo matching [5], multi-view reconstruction [6], and so on. Besides, some hybrid solutions have also been proposed [7]. 
An active approach can usually generate real-time depth data. The accuracy of depth data depends on the resolution of the sensor used. Comparing with modern CCD camera sensors, which can generally have 100 million or more pixels, most ToF cameras have much lower resolution. However, this kind of approach is still widely used in many scenarios. Newcombe et al. [8] used the Microsoft Kinect sensor to reconstruct an indoor scene. They employed a global optimization algorithm to estimate the camera's pose, and this algorithm could efficiently suppress the drift.

On the other hand, a passive approach takes a set of 2D images of the scene as input data, and extracts feature points from each image to match them. A 3D point cloud will be reconstructed from the matched correspondences. Snavely et al. [9] proposed a general pipeline of SfM. Some methods [10], [11] reconstruct the 3D scene model incrementally. On the other hand, other methods [12]-[14] optimize the parameters in a global scheme, which are usually faster than incremental methods. The reconstruction result generated by a SfM pipeline is a sparse 3D point cloud. It is difficult to extract the scene structure information from this sparse 3D point cloud. Furukawa and Ponce [15] proposed a patch based method to reconstruct a dense 3D point cloud. $\mathrm{Wu}$ [10], [16] proposed a useful tool, which combines the SfM pipeline and dense reconstruction algorithm together.

For a passive approach, it only needs a digital camera rather than some special sensors like in an active approach. The ubiquity of digital cameras today makes passive approaches very popular. In this paper, we focus on this kind of approach.

Many existing methods assume that the focal length of the camera can be extracted from either Exchangeable image file format (Exif) metadata of images or the precalibrated results. When camera is precalibrated, its zoom and focus settings must be fixed while taking photos. On the other hand, when a zoom lens is used, the Exif metadata are often used to extract the focal length. However, the extracted data are usually lack of accuracy. Some methods [17], [18] calibrate the camera on some selected positions, and estimate the focal length by using some interpolation methods. In this paper, we propose a new method to estimate the camera's focal length and principle point efficiently. This method takes some precalibrated results as seeds, and analyzes the changes of correspondences to calibrate the camera at an arbitrary position. Comparing with the linear interpolation method, our method can afford more accurate results. What is more important is that the whole calibration process can be made automatically.

One of the key problems when using a zoom lens is how to control the zoom lens properly. This is an important problem, because if a camera is zoomed out too much, a lot scene of details will be difficult to be detected from images. On the contrary, if it is zoomed in too much, the correspondences among images will be insufficient since only a small part of the scene has been captured. In this paper, we propose a criterion to control zoom lens properly. According to this criterion, users can easily capture the scene with sufficient details while avoiding the planar degeneracy.

Our system also includes a strategy for detecting and removing redundant photos. This strategy can speed up the computation of 3D reconstruction.

\section{Preliminaries}

In this section, the fundamental knowledge about 3D scene reconstruction using multi-view images is briefly explained. Please refer to [19] for more details.

\subsection{Epipolar Geometry and 2-View Reconstruction}

The simplest case is reconstructing the scene from only two views. The relationship between these two views is described by Epipolar geometry. Suppose that cameras $C_{1}$ and $C_{2}$ are viewing the same scene, and the corresponding image planes are $\pi_{1}$ and $\pi_{2}$ respectively (Fig. 1). Lights are reflected from a scene point to each camera center, which will hit on the image planes to construct its projections. 


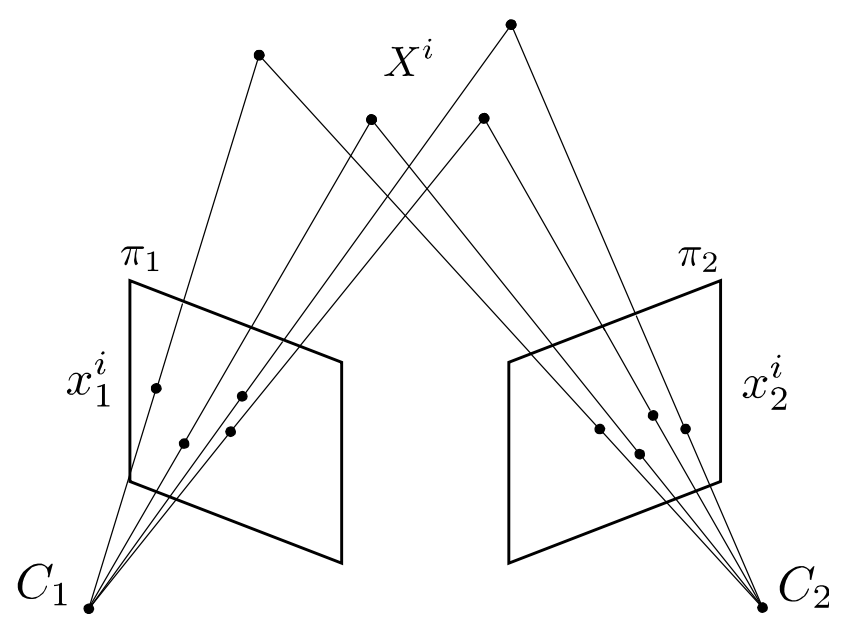

Fig. 1. Epipolar geometry.

For $i$-th scene point $X^{i}$, its projection on the $j$-th view is denoted as a 3 -element homogeneous vector $x_{j}^{i}$ :

$$
x_{j}^{i}=P_{j} X^{i},
$$

where, $P_{j}$ is a $3 \times 4$ homogeneous matrix, called projection matrix which has the following form:

$$
P_{j}=K_{j}\left[R_{j} \mid t_{j}\right]
$$

where, $K_{j}$ is called calibration matrix, $R_{j}$ is a $3 \times 3$ rotation matrix, $t_{j}$ is a 3 -element vector, and both of which describe the transformation from the world coordinate system to $j$-th camera coordinate system.

It is well known that for each pair of corresponding points, there exists the following equation:

$$
x_{2}^{i T} F x_{1}^{i}=0
$$

where, $x_{j}^{i}$ is the homogeneous image coordinate, $A^{T}$ denotes the transpose of matrix or vector $A, F$ is a $3 \times 3$ homogeneous matrix, called fundamental matrix. One known property of $F$ is:

$$
\operatorname{det}(F)=0,
$$

where, $\operatorname{det}(A)$ denotes the determinant of matrix $A$.

$F$ is a homogeneous matrix, so only the ratios among its elements are meaningful. Furthermore, $F$ is a singular matrix. Thus, $F$ has 7 degrees of freedom (DOF). One point pair can give one linear constraint about $F$, so at least 7 point pairs are necessary for estimating $F$ [20]. Besides, because the singularity property of $F$ will give a quadratic equation, which can have at most 4 roots (2 of which are imaginary), [21] describes an 8-point algorithm for estimating $F$.

When camera is calibrated, we can easily compute the normalized point as:

$$
\overline{x_{j}^{i}}=K_{j}^{-1} x_{j}^{i}
$$

where, $\overline{x_{j}^{i}}$ is a normalized point, and $K_{j}$ is the calibration matrix of $j$-th view. 
Similar to (3), there also exists a constraint between the normalized point pair:

$$
\overline{x_{2}^{i T}} E \overline{x_{1}^{i}}=0
$$

where, $E$ is a $3 \times 3$ homogeneous matrix called essential matrix with 5 DOF.

If we assume that the left camera coordinate system is aligned with the world coordinate system, its projection matrix will be:

$$
P_{1}=K_{1}[I \mid 0],
$$

where, $I$ is a $3 \times 3$ identity matrix, and 0 means a 3 -element zero vector. In this case, the projection matrix of the right view can be directly extracted from the essential matrix using singular value decomposition (SVD) as described in [19 pp. 258-259].

Therefore, we can construct simultaneous linear equations about $X^{i}$ by using (1), and reconstruct this scene point. Usually, the estimated 3D point $X^{i}$, as well as the projection matrix $P_{j}$, will be optimized by some nonlinear algorithms. This process is called bundle adjustment [22], [23].

\subsection{N-View Reconstruction}

$\mathrm{N}$-view reconstruction is an extension of 2-view reconstruction. As shown in Fig. 2, we can compute the camera's relative pose between each two adjacent views. After that, we need to combine all the reconstructed 3D points into one common coordinate system.

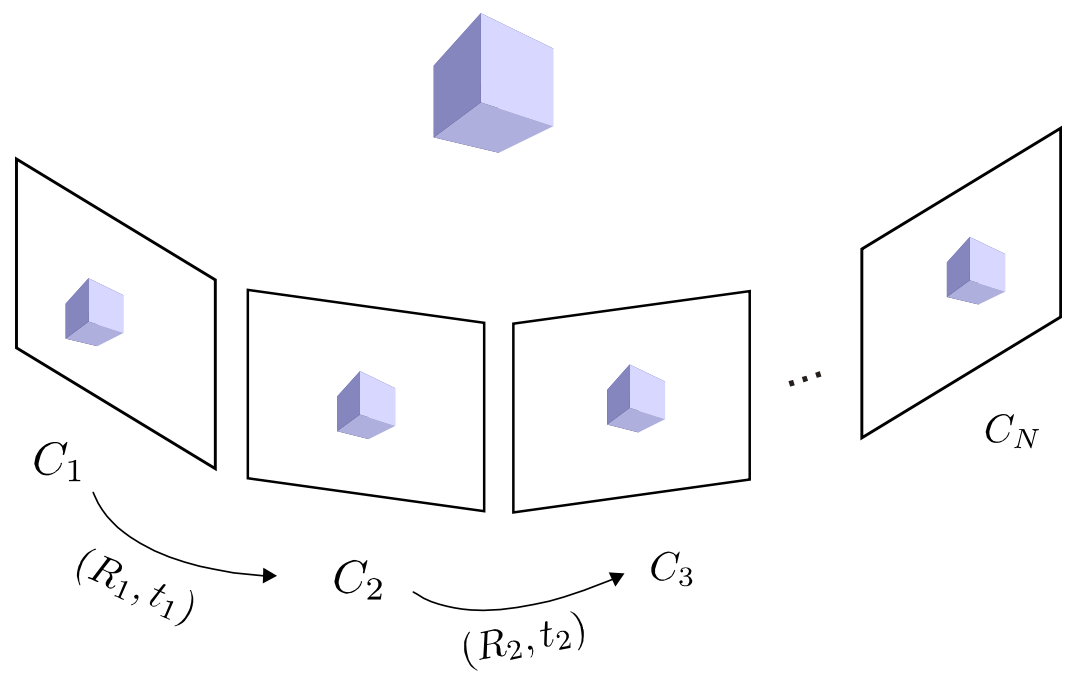

Fig. 2. N-view reconstruction.

Assume the reconstructed 3D point sets for image pairs $(1,2)$ and $(2,3)$ are $S_{12}$ and $S_{23}$ respectively. Notice that, these two sets may have different sizes. All members of $S_{12}$ are 3D points in the 1st camera coordinate system, whereas those in $S_{23}$ are in the 2nd camera coordinate system. We can apply a transformation on each point in $S_{12}$ :

$$
S_{12}^{i^{\prime}}=\left[\begin{array}{cc}
R_{1} & t_{1} \\
0 & 1
\end{array}\right] S_{12}^{i} .
$$

As a result, each point in set $\left\{S_{23} \cup S_{12}^{\prime}\right\}$ is in the 2 nd camera coordinate system. 
Similar transformations can be applied to other image pairs. Finally, all the reconstructed 3D points are aligned into a common coordinate system to form a 3D point cloud.

\subsection{Dense 3D Reconstruction}

Usually, the result of section 2.2 is a sparse 3D point cloud, from which it is very difficult to understand the structure of the scene. Thus, we need to utilize some algorithms to densify the point cloud. One of the state-of-the-art dense 3D reconstruction methods is [15]. Here, we give a brief introduction of [15], please refer to the original work for more details.

At first, features are detected by Harris and Difference-of-Gaussian (DoG) operators, and are matched along the Epipolar line. The matched features are then reconstructed as a small patch in the scene. This step is called the matching phase. The second step is an expansion, in which the initial reconstructed patches are spread to nearby pixels to obtain more patches. In the third step, which is called filtering, the visibility constraint is checked for each patch to eliminate the outliers lying either in front or behind the observed surface. The expansion and filtering steps are repeated for several times until no more patches can be added. Usually, the expansion step is the most time consuming step, and it may take several minutes to few hours according to the size of input image data.

\section{Proposed Solution}

In this section, the proposed binocular vision system is explained. This system uses our new calibration method to estimate camera's focal length. The details about this calibration method are discussed below. We also explain the criterion for controlling zoom lens, and the strategy for checking redundant images.

\subsection{Binocular Vision System}

Fig. 3 shows our proposed binocular vision system. It consists of three main parts: two pan-tilt-zoom (PTZ) cameras, a notebook, and a robot.

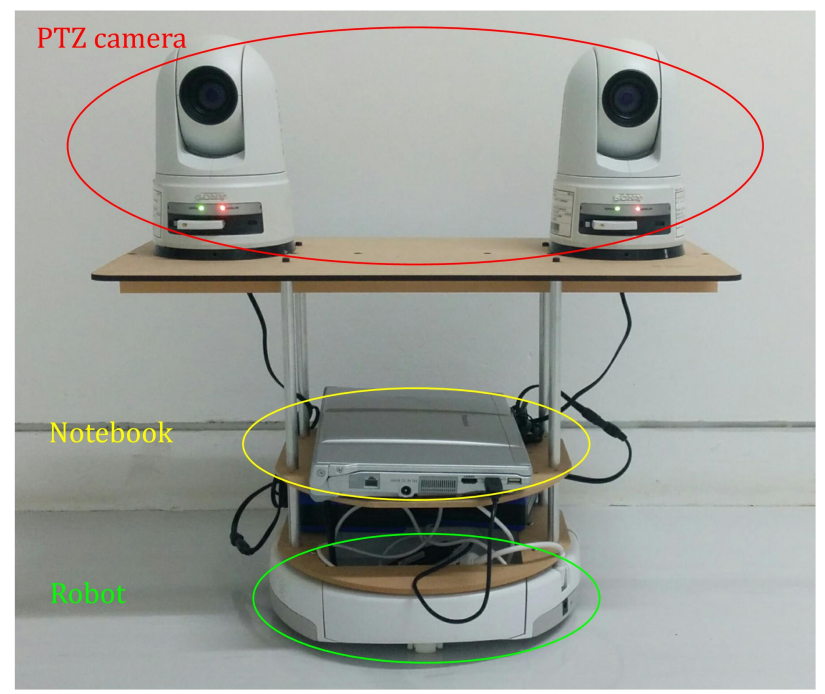

Fig. 3. Proposed binocular vision system.

Cameras are used to perceive the scene. We use two SONY SNC-RZ25N cameras, which provide Http commands to download the image, and control camera's pan, tilt, zoom and focus positions. They also have a network function that enables remote control.

Cameras are mounted on a platform, which is equipped on a programmable robot, iRobot Create 4400 . This robot has a serial port for communication, which is converted to a USB interface. This USB is plugged to 
a notebook. We installed a tool [24] on this notebook to control the robot.

A remote PC can move the robot by controlling the notebook, and retrieve image frames from cameras through network. Our system will then analyze the image and give suggestions to help users to control the zoom lens. Once users decide to capture the scene, the current frame will be checked by our proposed redundancy checking strategy. Once it passes the check, this image and camera settings will be written to disk. Then, the camera will be calibrated by using the proposed calibration method. When this is done, it is ready to move the robot and capture other photos.

\subsection{Zoom Lens Calibration}

Camera calibration is to estimate the camera calibration matrix, $K$, which has the following form:

$$
K=\left[\begin{array}{ccc}
f_{u} & s & u_{0} \\
0 & f_{v} & v_{0} \\
0 & 0 & 1
\end{array}\right]
$$

where, $f_{u}$ and $f_{v}$ are focal lengths on horizontal and vertical axes respectively, $s$ is called skew parameter, and $\left(u_{0}, v_{0}\right)$ is called a principle point. Note that, in some cases, the lens distortion coefficients are also estimated during the calibration. However, for simplicity of explanation, we assume the lens distortion is negligible. For most modern cameras, it is reasonable to assume that $f_{u}=f_{v}, s=0$ and $\left(u_{0}, v_{0}\right)$ is located at the image center [25], [26].

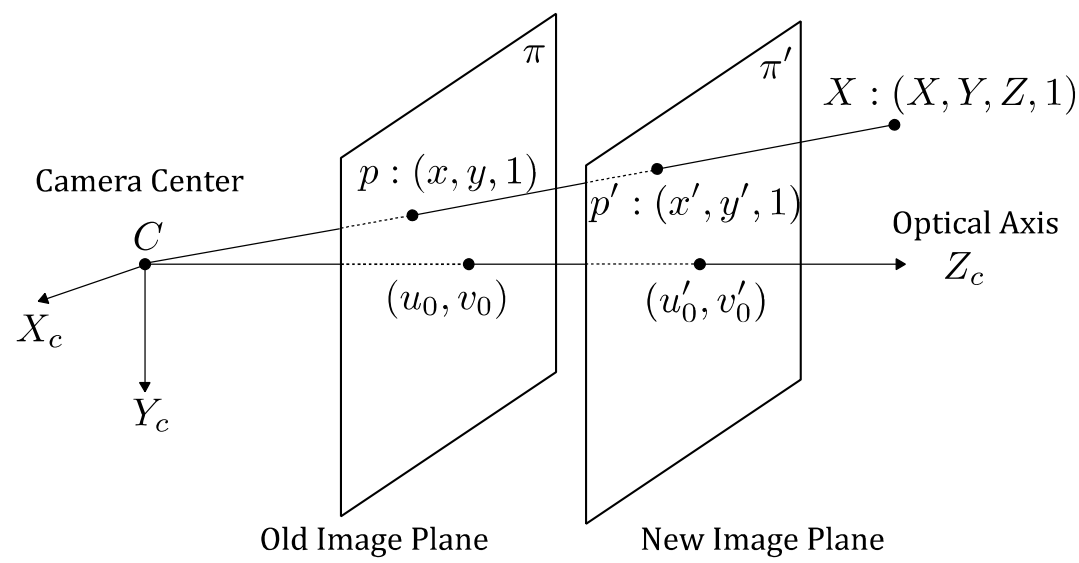

Fig. 4. Change focal length in Pinhole camera model.

For a camera with a zoom lens, changing zoom or focus will result in changes of focal length in the Pinhole camera model. As shown in Fig. 4, this is equivalent to move the image plane along the optical axis to a new place. The geometric meaning of camera's focal length is the distance from camera center to the intersection of its image plane and optical axis. Thus, one can easily derive the following equation:

$$
r_{f}=r_{u}=r_{v}, r_{f}=\frac{f^{\prime}}{f}, r_{u}=\frac{x^{\prime}-u_{0}^{\prime}}{x-u_{0}}, r_{v}=\frac{y^{\prime}-v_{0}^{\prime}}{x-v_{0}}
$$

where, $f$ and $f^{\prime}$ are the old and new focal lengths, respectively.

In real world experiments, because of unavoidable errors in locations of points, $r_{u}$ and $r_{v}$ usually take different values. Furthermore, if we choose another point pair, the result will be different. In addition, due to imperfections of lens manufacturing, the optical axis will move slightly after changing zoom or focus, which means $u_{0} \neq u_{0}^{\prime}$ and $v_{0} \neq v_{0}^{\prime}$. Thus, we propose an energy function as: 


$$
F\left(u_{0}, v_{0}, u_{0}^{\prime}, v_{0}^{\prime}\right)=\sqrt{\frac{1}{2 N} \sum_{i=1}^{N}\left(\left(r_{u}^{i}-\mu_{r}\right)^{2}+\left(r_{v}^{i}-\mu_{r}\right)^{2}\right)},
$$

with

$$
\mu_{r}\left(u_{0}, v_{0}, u_{0}^{\prime}, v_{0}^{\prime}\right)=\frac{1}{2 N} \sum_{i=1}^{N}\left(r_{u}^{i}+r_{v}^{i}\right)
$$

where, $N$ is the number of point pairs matched between the old and new views.

Concretely speaking, the value of (11) is the standard deviation of $r_{u}$ and $r_{v}$ for a given parameter set $\left(u_{0}, v_{0}, u_{0}^{\prime}, v_{0}^{\prime}\right)$. By minimizing this energy, we can find the optimal locations of $\left(\hat{u}_{0}, \hat{v}_{0}\right)$ and $\left(\hat{u}_{0}^{\prime}, \hat{v}_{0}^{\prime}\right)$. As a result, the new focal length can be computed as:

$$
f^{\prime}=\hat{\mu}_{r} \cdot f
$$

The old focal length is either precalibrated by some calibration tools, or the result of previous calibration by using our method. In order to suppress accumulative errors, we suggest taking several precalibrated results as seeds for our method.

\subsection{Control of Zoom Lens}

When we use a zoom lens, one key question is: how shall we set the zoom at a proper position? Thus, we need a criterion to determine whether the current zoom position is proper or not. A proper zoom position should at least satisfy that scene details are clear enough to be detected in the image, and that the current image will be used in the 3D reconstruction. Based on this analysis, we define two terms for controlling a zoom lens properly.

The first term is called feature density (FD) of view $I$, defined as:

$$
F D(I)=\frac{\text { number of detected features in } I}{\text { number of all pixels in I }}
$$

When feature density is higher than a predefined threshold $\tau_{h}$, the current shooting area probably contains a lot of textures. In this case, it is better to zoom in further to capture more details of the scene. During this operation, the feature density may become higher because more scene textures can be detected. However, the feature density will start getting lower at some point. This is because the current zoom is sufficient enough comparing with the richness of scene texture. Thus, we should stop zooming in any further if feature density becomes lower than a predefined threshold $\tau_{l}$.

The second term is called degree of planar degeneracy (DPD), which measures the unlikeness of the current shooing area is a planar object. For a pair of views, if all the correspondences lie on a plane in the real world, then there exists a 2D transformation between these two views:

$$
x_{2}^{i}=H x_{1}^{i}
$$

where, $H$ is a $3 \times 3$ homogeneous matrix.

We can substitute (15) for (3), and get:

$$
x_{1}^{i T} H^{T} F x_{1}^{i}=0
$$


One can verify that for any $3 \times 3$ skew symmetric matrix $M$, the following relationship holds:

$$
x_{1}^{i T} M x_{1}^{i}=0 .
$$

Thus, the fundamental matrix $F$ can be computed as:

$$
F=H^{-T} M
$$

Therefore, $F$ cannot be uniquely defined. Consequently, it is impossible to uniquely determine the camera projection matrix, and the 3D positions of corresponding points. This is a degenerated case which should be avoided. The degree of planar degeneracy is defined as the average reprojection error of $H$ :

$$
D P D\left(I_{1}, I_{2}\right)=\frac{1}{N} \sum_{i=1}^{N} \operatorname{dis}\left(H x_{1}^{i}, x_{2}^{i}\right)
$$

where, $\operatorname{dis}(a, b)$ measures the Euclidean distance between points $a$ and $b$.

When the degree of planar degeneracy is smaller than a predefined threshold $\delta$, it means the matching points probably lie on a plane. Thus, we should zoom out the lens in order to avoid the planar degeneracy. On the other hand, when the degree of planar degeneracy is bigger than the threshold, it implies that the current shooting area is not like a plane. In this case, it is sensible to zoom in further if necessary.

In a summary, our criterion for controlling the zoom lens is:

$$
\text { zoom suggestion } \begin{cases}\text { zoom in, } & \text { if } F D>\tau_{h} \text { and } D P D>\delta \\ \text { zoom out, } & \text { if } F D<\tau_{l} \text { or } D P D<\delta . \\ \text { proper, } & \text { otherwis }\end{cases}
$$

The user should adjust the zoom lens gradually according to this suggestion, until our system gives judgement of "proper".

\subsection{Strategy for Reducing Redundant Images}

In order to reconstruct the 3D model of a scene, one needs to take many photos from different viewpoints. However, if the input image set is too big, the computation time of 3D reconstruction will become very long. In this paper, we propose a strategy to detect and reject redundant images to speed up the computation.

Suppose an image set $S$, an image $I$ that does not belong to $S$, feature points detected on $I$ are denoted as $x_{i}$. We match image $I$ with each image in $S$, and define a counter $c_{i}$ for each feature point $x_{i}$ to record its matched times. For example, if $x_{i}$ is matched with another feature in an image from $S$, then $c_{i}$ will be increased by 1 . After matching with all images in $S$, we can label each feature point in $I$ as:

$$
\text { label of } x_{i}= \begin{cases}\text { redundant, } & \text { if } c_{i} \geq \gamma_{N} \\ \text { matched, } & \text { if } 0<c_{i}<\gamma_{N} \\ \text { nonmatched, } & \text { if } c_{i}=0\end{cases}
$$

where, $\gamma_{N}$ is a predefined threshold.

Then, we compute the portion of features labeled as "redundant". If this portion is too big, it means most of features in $I$ have already been found in $S$. In other words, $I$ can only give limited contributions even if added to $S$. Thus, we will reject image $I$ if its portion of redundant features exceeds a threshold $\gamma_{p}$.

Furthermore, in real world scenarios, it may take a long time to match each image in set $S$. Instead, we can 
randomly select $\gamma_{C}$ images in $S$.

Before saving the current view, our system will automatically check its degree of redundancy. Only when this view passes the check, it will be added to the image set used for 3D reconstruction.

\section{Experimental Results}

We conducted two experiments to verify the proposed vision system. The first experiment is to demonstrate the performance of our new camera calibration method. The second one is to test the efficiency of the proposed zoom lens control criterion, and the redundant image checking strategy.

\subsection{Zoom Lens Calibration Experiment}

In this experiment, we compared our proposed calibration method with a general linear interpolation method. As mentioned before, the Sony SNC-RZ25N camera was used. The camera's zoom position can be moved freely in range of $[0,16000]$ (encoder value without unit).

We calibrated the camera at positions 0 to 16000 with step of 1000 using the Mat lab Computer Vision System Toolbox [27]-[29]. These calibration results were taken as ground truth data.

Two cases were tested in this experiment. Firstly, ground truth data at positions 0 to 16000 with step of 4000 were taken as the seeds to each calibration method. Secondly, positions 0 to 16000 with a smaller step, 2000, were taken as the seeds data. In both cases, the two methods were evaluated at positions 1000 to 15000 with step of 1000 (if it was not included in the seeds data). The absolute relative error (ARE) defined as the following equation was measured for each test case:

$$
A R E=\frac{f-f^{\prime}}{f} \mid
$$

where, $f$ and $f^{\prime}$ are the ground truth and estimated focal length, respectively. Notice that, the toolbox could produce two focal length values on horizontal and vertical axes individually. Their average was taken as a ground truth.
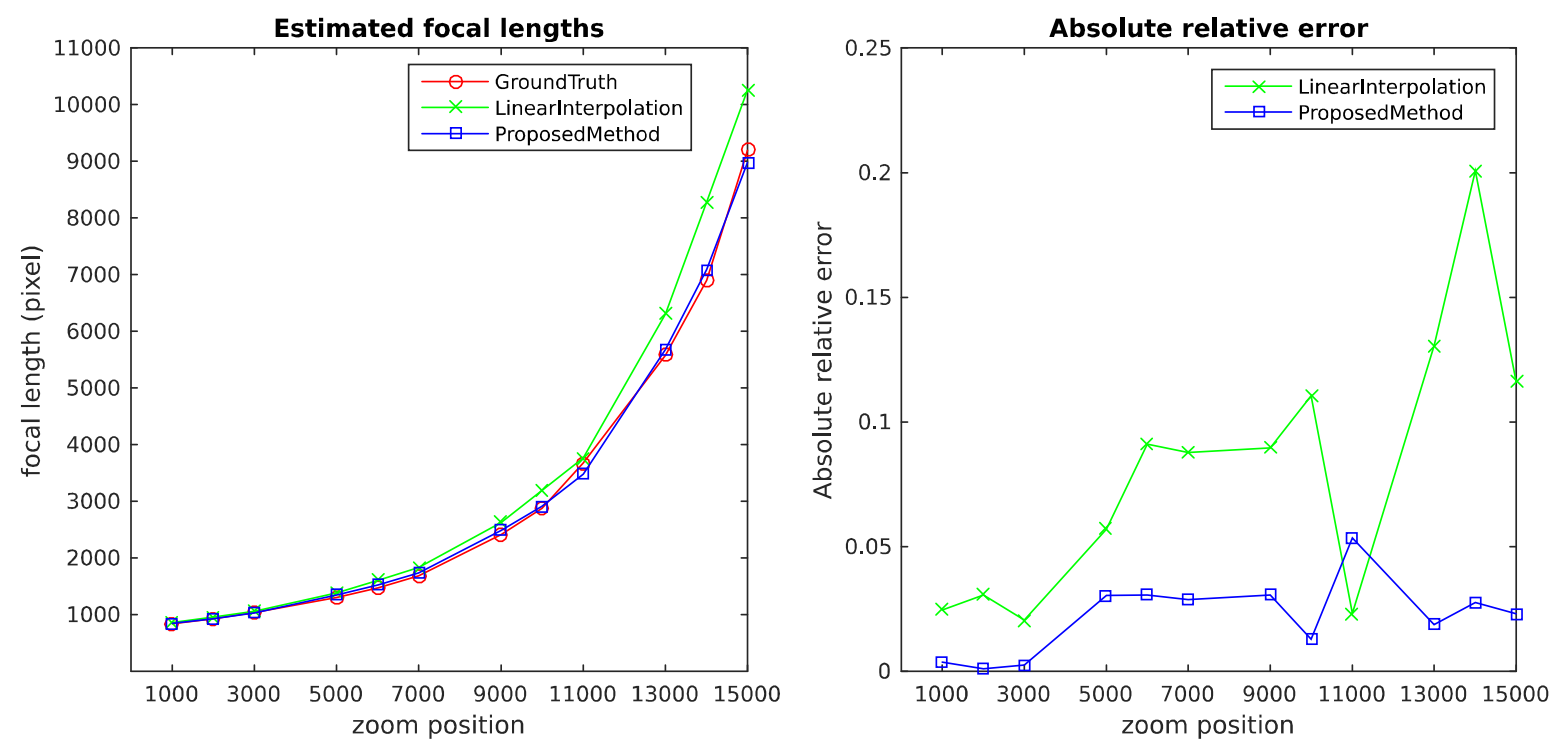

Fig. 5. Results when step of seed data is 4000 (first case).

As shown in Fig. 5 and 6, the camera's focal length changes slowly in range 0 to 6000 . According to the camera's data sheet, the zoom ratio only changes to about $2 \times$ at position 6000 . When zoom lens is moved 
within this range, the linear interpolation method can approximate the focal length very well. On the other hand, our proposed method also could produce accurate estimations. Obviously, we can easily know that our method outperformed the linear interpolation method in this range by comparing their AREs.

The zoom ratio changes from about $2 \times$ to $4 \times$ when zoom is moved from 6000 to 10000 . The camera's focal length changes a little more rapidly in this range. From the right side of Fig. 5 and Fig. 6, we can clearly see that the AREs of each method became bigger. As in the previous range, our proposed method could produce much more accurate results than the linear interpolation in all cases of this range.

The camera's focal length changes very fast when zoom position is bigger than 10000 . In fact, the corresponding zoom ratios for positions 10000 and 16000 are about $4 \times$ and $18 \times$, respectively. Thus, estimating the focal length becomes more challenging in this range. The AREs of both methods in this range were much bigger than those of other ranges. In most cases, our proposed method could still outperform the linear interpolation method.
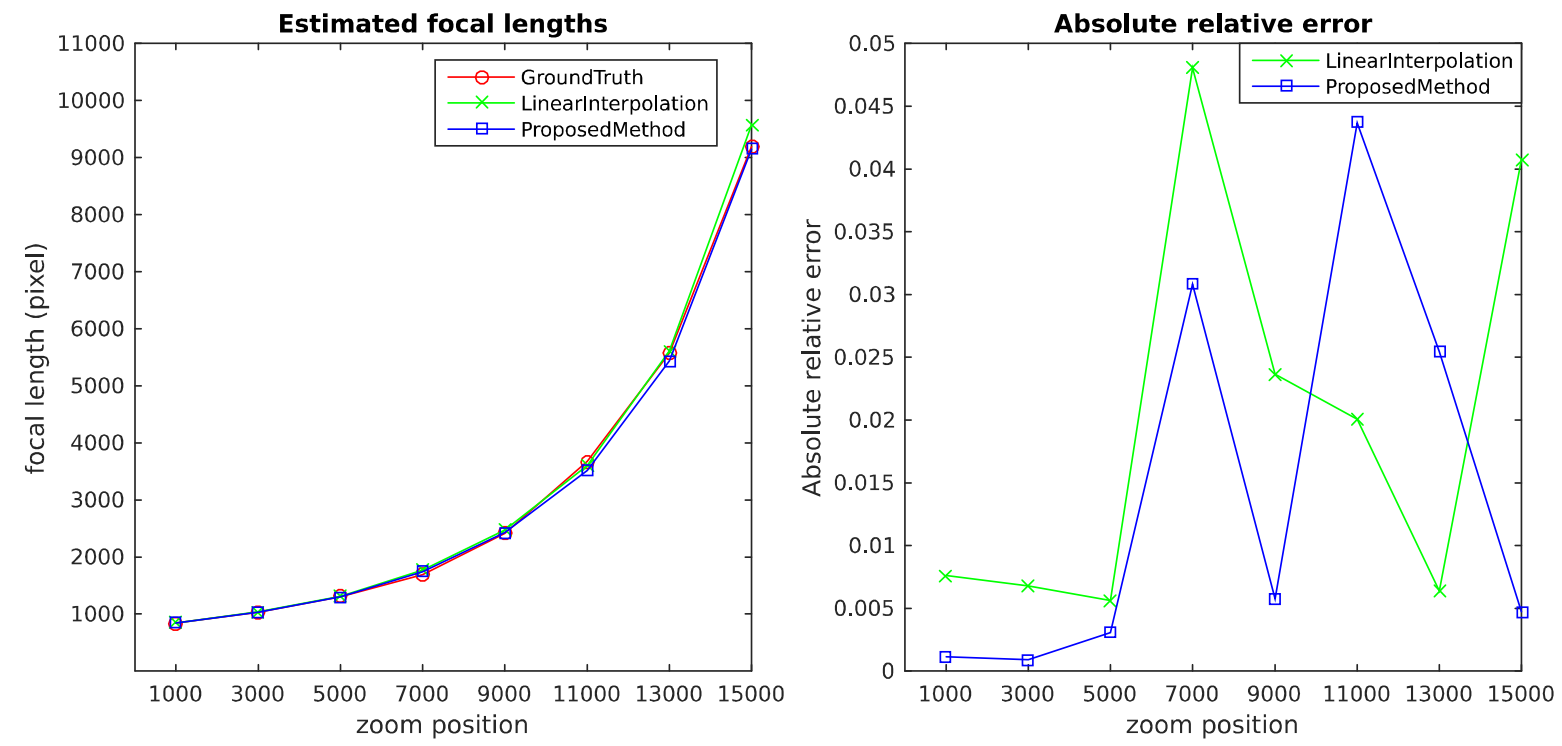

Fig. 6. Results when step of seed data is 2000 (second case).

From Fig. 5 to Fig. 6, we can see that the AREs of each method became smaller. This is because that the step of seed positions changes from 4000 to 2000. Thus, more accurate results could be expected.

The average AREs over all tested positions for the linear interpolation method are 0.0819 and 0.0199 when the step of seed positions are 4000 and 2000, respectively. The averages AREs of our proposed method are 0.0219 and 0.0144 for seed steps 4000 and 2000, respectively. Thus, it can be concluded that our method could generate more accurate results than the linear interpolation method.

Our method is advantageous when comparing with the interpolation method. For a zoom lens, the focal length will change if either zoom or focus setting is changed. Thus, strictly speaking, one needs construct a 2D interpolant which takes the combination of zoom and focus positions as index. It is a time consuming work to collect calibration results to construct such an interpolant. However, our method can dynamically calibrate the camera on any focus or zoom position. The only constraint of our method is that the current shooting area should not be textureless, which can be satisfied easily.

\subsection{D Scene Reconstruction Experiment}

The goal for this experiment is to verify the proposed zoom lens control criterion, and image redundancy checking strategy. OpenMVG [30] was used for sparse reconstruction, and OpenMVS [31] was used for dense reconstruction and mesh estimation. Parameters for each test case are listed in Table 1. 
Table 1. Parameter Settings for Each Test Case

\begin{tabular}{|l|l|l|l|}
\hline Case No. & Name & Zoom Control & Redundancy Checking \\
\hline 1 & Fixed Focal & fixed zoom and focus & None \\
\hline 2 & Fixed Focal More & fixed zoom and focus & None \\
\hline 3 & Checked Fixed Focal & fixed zoom and focus & proposed strategy \\
\hline 4 & Zoom & proposed criterion & None \\
\hline 5 & Zoom More & Proposed criterion & None \\
\hline 6 & Checked Zoom & proposed criterion & proposed strategy \\
\hline
\end{tabular}

In cases 1, 2 and 3, we fixed the zoom and focus settings at the same position during experiment. In case 4, 5 and 6, we adjusted the zoom lens according to the suggestions given by the proposed zoom control criterion. In cases 1, 2, 4 and 5, the redundancy checking function was disabled. In cases 3 and 6 , we checked the redundant views by using the proposed strategy. Case 2 is similar to case 1 , except that more photos were taken and used for reconstruction. This scheme is also used for cases 4 and 5. For all cases, the parameters used by our proposed zoom control criterion and redundancy checking strategy (if enabled) are listed in Table 2.

Table 2. Parameters for Proposed Zoom Control Criterion and Redundancy Checking Strategy

\begin{tabular}{|l|l|l|}
\hline Name & Value & Meaning \\
\hline$\tau_{l}$ & 0.1 & Feature density lower threshold \\
\hline$\tau_{h}$ & 0.3 & Feature density upper threshold \\
\hline$\delta$ & 0.5 & Degree of planar degeneracy threshold \\
\hline$\gamma_{C}$ & 10 & Number of views used for redundancy checking \\
\hline$\gamma_{N}$ & 5 & Redundancy checking threshold \\
\hline$\gamma_{p}$ & 0.2 & Max portion of redundant features \\
\hline
\end{tabular}

This experiment was conducted on a Dell Precision T5610 PC with an Intel Xeon E5-2609 v2 CPU (2.50 GHz x 8 cores) and 32 GB memory. Table 3 lists the sparse, dense and mesh reconstruction results for each case. The final 3D models, as well as some selected parts are shown in Fig. 7. The corresponding test cases from top to bottom rows in Fig. 7 are cases 1 to 6 .

Table 3. Results for Each Test Case

\begin{tabular}{|c|c|c|c|c|c|c|}
\hline $\begin{array}{l}\text { Case } \\
\text { No. }\end{array}$ & $\begin{array}{l}\text { Number of } \\
\text { Vertices after } \\
\text { sparse } \\
\text { reconstruction }\end{array}$ & $\begin{array}{l}\text { Number of } \\
\text { Vertices after } \\
\text { dense } \\
\text { reconstruction }\end{array}$ & $\begin{array}{l}\text { Number of } \\
\text { Vertices after } \\
\text { mesh refinement }\end{array}$ & $\begin{array}{l}\text { Number of } \\
\text { Faces after } \\
\text { mesh } \\
\text { refinement }\end{array}$ & $\begin{array}{l}\text { Number of } \\
\text { Images } \\
\text { used }\end{array}$ & $\begin{array}{l}\text { Total } \\
\text { computation } \\
\text { time } \\
\text { (second) }\end{array}$ \\
\hline 1 & 1581 & 1290562 & 20677 & 41220 & 40 & 602 \\
\hline 2 & 1531 & 1333302 & 17741 & 35320 & 70 & 693 \\
\hline 3 & 1321 & 900152 & 18094 & 36025 & 31 & 309 \\
\hline 4 & 2578 & 1302996 & 34283 & 68256 & 63 & 515 \\
\hline 5 & 3379 & 1926259 & 41608 & 82890 & 80 & 750 \\
\hline 6 & 1837 & 872722 & 22985 & 45664 & 53 & 432 \\
\hline
\end{tabular}



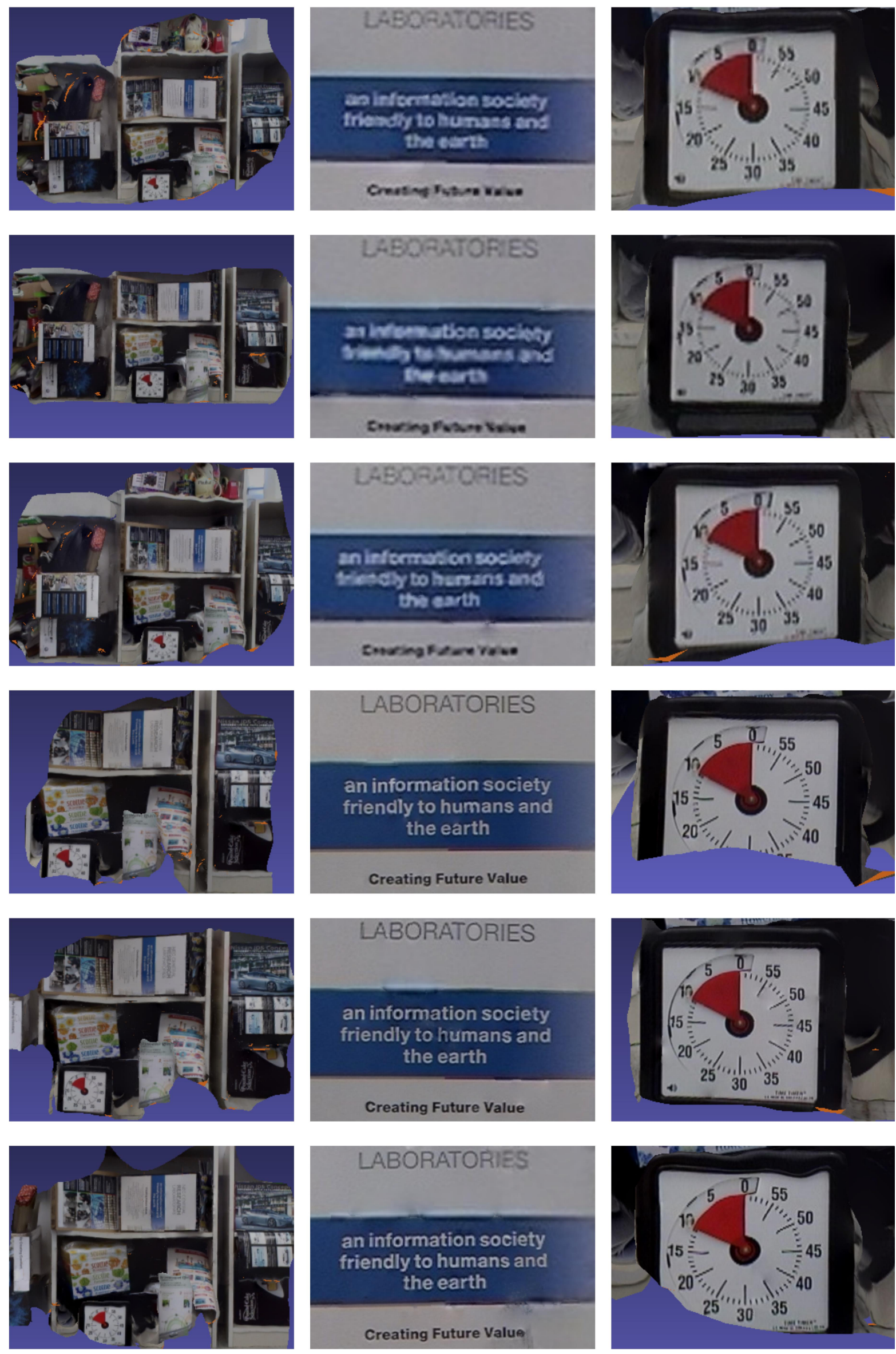

Fig. 7. Reconstruction results of test cases 1 to 6 (from top to bottom). 
As shown in Fig. 7, there is no significant difference between the results of cases 1 and 2. Even more images were given and more time were used for computation, the improvements from case 1 to 2 were very limited. On the contrary, the quality of some parts of the scene even became worse. For example, in the second column of Fig. 7, the texts are more unrecognizable in case 2 than in case 1. In the third column of Fig. 7, the numbers on the clock are blurred more severely in case 2 than case 1 . Similarly, the improvements of reconstruction quality from case 4 to 5 were also very limited. However, unlike cases 1 and 2 , it seemed that no part of the scene became more blurred.

This result implies the interesting fact that more images could not guarantee better result. We think the reason is that the quality of reconstruction depends most on the clarity of scene details in images. Though the number of images increased, the clarity of them remained the same.

Obviously, the overall reconstruction results when using a zoom lens (cases 4 to 6) are better than using a fixed focal lens (cases 1 to 3). For example, the texts in the second column of Fig. 7 are much more readable in cases 4 and 5, whereas they are severely blurred in cases 1 and 2. The numbers in the third column of Fig. 7 are also much clearer in cases 4 and 5 than in cases 1 and 2. This result implies that the proposed criterion can help users to control the zoom lens properly, and to take photos with better clarity of details.

Although using more images could not improve the quality of reconstruction, it could afford better completeness. The first column of Fig. 7 is an overview of the reconstructed 3D model. It can be seen clearly that cases 2 and 5 are more complete than cases 1 and 4, respectively.

The reconstruction quality of case 3 is almost the same as case 1 . In case 3 , our proposed strategy was used to detect and reject redundant images. Thus, a smaller input image set was used in case 3 than case 1 . Similarly, this redundancy checking function was disabled in case 4 , whereas enabled in case 6 . There was no obvious drop in reconstruction quality after enabling our redundancy checking strategy.

As shown in Table 3, our proposed redundancy checking strategy resulted in a decrease in computation time. For the fixed focal lens cases ( 1 and 3 ), the computation time was reduced at about $50 \%$. The computation time was reduced at about $16 \%$ for the zoom lens cases ( 4 and 6 ).

In a summary, our proposed zoom control criterion could help users control the zoom lens properly, and take photos with clearer scene details. This could improve the reconstruction quality. On the other hand, our redundancy checking strategy could detect and reject redundant images efficiently, and speeded up the computation. The reconstruction quality after applying this strategy remained almost the same.

\section{Conclusion}

In this paper, we proposed an intelligent binocular vision system with zoom lens for reconstructing the 3D model of a scene. This system can dynamically calibrate the camera by using the proposed calibration method. This method can give more accurate results than the linear interpolation method. We also proposed a criterion to help users control the zoom lens properly. According to this criterion, users can easily take photos with clear scene details while avoiding the planar degeneracy. A strategy for detecting redundant images was also presented. Experimental results showed that the quality of reconstructed 3D models remained almost the same after applying the redundancy checking strategy, while the computation time reduced a lot. In future, we are planning to improve our vision system to be more intelligent, which can automatically reconstruct the scene without or with few user instructions.

\section{References}

[1] Civera, J., Grasa, O. G., Davison, A. J., \& Montiel, J. M. M. (2010). 1-Point RANSAC for extended Kalman filtering: Application to real-time structure from motion and visual odometry. Journal of Field Robotics, 27(5), 609-631. 
[2] Engel, J., Schöps, T., \& Cremers, D. (2014, September). LSD-SLAM: Large-scale direct monocular SLAM. European Conference on Computer Vision (pp. 834-849). Springer International Publishing.

[3] Choi, J. (2014, October). Hybrid map-based SLAM using a Velodyne laser scanner. 17th International IEEE Conference on Intelligent Transportation Systems (ITSC) (pp. 3082-3087). IEEE.

[4] Hochdorfer, S., \& Schlegel, C. (2010, October). 6 DoF SLAM using a ToF camera: The challenge of a continuously growing number of landmarks. Intelligent Robots and Systems (IROS), 2010 IEEE/RSJ International Conference on (pp. 3981-3986). IEEE.

[5] Banz, C., Hesselbarth, S., Flatt, H., Blume, H., \& Pirsch, P. (2010, July). Real-time stereo vision system using semi-global matching disparity estimation: Architecture and FPGA-Implementation. Embedded Computer Systems (SAMOS), 2010 International Conference on (pp. 93-101). IEEE.

[6] Wu, T. P., Yeung, S. K., Jia, J., \& Tang, C. K. (2010, June). Quasi-dense 3D reconstruction using tensor-based multiview stereo. Computer Vision and Pattern Recognition (CVPR), 2010 IEEE Conference on (pp. 1482-1489). IEEE.

[7] Castaneda, V., Mateus, D., \& Navab, N. (2011, January). SLAM combining ToF and high-resolution cameras. Applications of Computer Vision (WACV), 2011 IEEE Workshop on (pp. 672-678). IEEE.

[8] Newcombe, R. A., Izadi, S., Hilliges, O., Molyneaux, D., Kim, D., Davison, A. J., Kohli, P., Shotton, J., Hodges, S. \& Fitzgibbon, A. (2011, October). KinectFusion: Real-time dense surface mapping and tracking. Mixed and Augmented Reality (ISMAR), 2011 10th IEEE International Symposium on (pp. 127-136). IEEE.

[9] Snavely, N., Seitz, S., \& Szeliski, R. (2006). Photo tourism: Exploring image collections in 3D. ACM transactions on graphics. ACM Transactions on Graphics.

[10] Wu, C. (2013, June). Towards linear-time incremental structure from motion. 2013 International Conference on 3D Vision-3DV 2013 (pp. 127-134). IEEE.

[11] Klopschitz, M., Irschara, A., Reitmayr, G., \& Schmalstieg, D. (2010). Robust incremental structure from motion. Proc. 3DPVT (2) 1-8.

[12] Jiang, N., Cui, Z., \& Tan, P. (2013). A global linear method for camera pose registration. Proceedings of the IEEE International Conference on Computer Vision (pp. 481-488).

[13] Moulon, P., Monasse, P., \& Marlet, R. (2013). Global fusion of relative motions for robust, accurate and scalable structure from motion. Proceedings of the IEEE International Conference on Computer Vision (pp. 3248-3255).

[14] Wilson, K., \& Snavely, N. (2014, September). Robust global translations with 1dsfm. European Conference on Computer Vision (pp. 61-75). Springer International Publishing.

[15] Furukawa, Y., \& Ponce, J. (2010). Accurate, dense, and robust multiview stereopsis. IEEE Transactions on Pattern Analysis and Machine Intelligence, 32(8), 1362-1376.

[16] Wu, C., Agarwal, S., Curless, B., \& Seitz, S. M. (2011, June). Multicore bundle adjustment. Computer Vision and Pattern Recognition (CVPR), 2011 IEEE Conference on (pp. 3057-3064). IEEE.

[17] Kim, M. Y., Ayaz, S. M., Park, J., \& Roh, Y. (2014). Adaptive 3D sensing system based on variable magnification using stereo vision and structured light. Optics and Lasers in Engineering, 55, 113-127.

[18] Liu, P., Willis, A., \& Sui, Y. (2009, February). Stereoscopic 3D reconstruction using motorized zoom lenses within an embedded system. IS\&T/SPIE Electronic Imaging (pp. 72510W-72510W). International Society for Optics and Photonics.

[19] Hartley, R. I., \& Zisserman, A. (2004). Multiple view geometry in computer vision (2nd ed.). Cambridge: university press.

[20] Hartley, R. I. (1994). Projective reconstruction and invariants from multiple images. IEEE Transactions on Pattern Analysis and Machine Intelligence, 16(10), 1036-1041.

[21] Longuet-Higgins, H. C. (1987). A computer algorithm for reconstructing a scene from two projections. 
Readings in Computer Vision: Issues, Problems, Principles, and Paradigms, MA Fischler and O. Firschein, eds, 61-62.

[22] Triggs, B., McLauchlan, P. F., Hartley, R. I., \& Fitzgibbon, A. W. (1999, September). Bundle adjustment-a modern synthesis. International Workshop on Vision Algorithms (pp. 298-372). Springer Berlin Heidelberg.

[23] Lourakis, M. I., \& Argyros, A. A. (2009). SBA: A software package for generic sparse bundle adjustment. ACM Transactions on Mathematical Software (TOMS), 36(1), 2.

[24] Kruesi, D., \& Grob, D. (2009). Emss iRobot create framework. Retrieved September 18, 2016, from http://emssframework.sourceforge.net

[25] Dou, S., Nagahashi, H., \& Zhang, X. (2014, January). An efficient solution to 3D reconstruction from two uncalibrated views under SV constraint. Computer Vision Theory and Applications (VISAPP), 2014 International Conference on, 3, 664-671. IEEE.

[26] Pernek, Á., \& Hajder, L. (2013). Automatic focal length estimation as an eigenvalue problem. Pattern Recognition Letters, 34(9), 1108-1117.

[27] The MathWorks, Inc. (2016) Computer Vision System Toolbox. Retrieved September 18, 2016, from http://www.mathworks.com/help/vision/index.html

[28] Zhang, Z. (2000). A flexible new technique for camera calibration. IEEE Transactions on Pattern Analysis and Machine Intelligence, 22(11), 1330-1334.

[29] Heikkila, J., \& Silvén, O. (June 1997). A four-step camera calibration procedure with implicit image correction. 1997 IEEE Computer Society Conference on Computer Vision and Pattern Recognition (pp. 1106-1112). IEEE.

[30] Moulon, P., Monasse, P., \& Marlet, R. (2016). OpenMVG (open Multiple View Geometry). Retrieved September 18, 2016, from https://github.com/openMVG/openMVG

[31] OpenMVS (open Multi-View Stereo reconstruction library). Retrieved September 18, 2016, from https://github.com/cdcseacave/openMVS/wiki

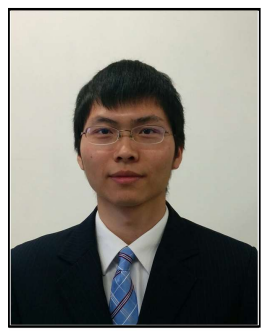

Shuyang Dou received his BS degree in software engineering from Dalian University of Technology in 2009, and MS degree from Tokyo Institute of Technology in 2013, respectively. He is currently a doctoral student at Tokyo Institute of Technology. His main research interests include camera calibration, 3D reconstruction, texture mapping and stereo matching.

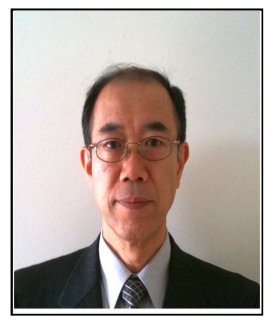

Hiroshi Nagahashi is with Faculty of Laboratory for Future Interdisciplinary Research of Science and Technology (FIRST), Tokyo Institute of Technology. He received his BS, MS and PhD degrees from Tokyo Institute of Technology in 1975, 1977 and 1980, respectively. His research work focuses on 3D geometric modeling, machine learning, medical image processing and artificial intelligence. 